\title{
The DnaJ-Like Zinc Finger Domain Protein PSA2 Affects Light Acclimation and Chloroplast Development in Arabidopsis thaliana
}

\author{
Yan-Wen Wangt, Si-Ming Chent, Wei-Jie Wang, Xing-Qi Huang, Chang-Fang Zhou, \\ Zhong Zhuang and Shan Lu*
}

State Key Laboratory of Pharmaceutical Biotechnology, School of Life Sciences, Nanjing University, Nanjing, China

OPEN ACCESS

Edited by:

Jirong Huang,

University of Tokyo, Japan

Reviewed by:

Yan Lu,

Western Michigan University, USA

Li Li,

Cornell University, USA

*Correspondence:

Shan Lu

shanlu@nju.edu.cn

tThese authors have contributed equally to this work.

Specialty section: This article was submitted to Plant Physiology, a section of the journa Frontiers in Plant Science

Received: 08 February 2016 Accepted: 08 March 2016 Published: 24 March 2016

Citation Wang $Y-W$, Chen S-M, Wang W-J, Huang $X-Q$, Zhou $C-F$, Zhuang $Z$ and Lu S (2016) The DnaJ-Like Zinc Finger Domain Protein PSA2 Affects

Light Acclimation and Chloroplast Development in Arabidopsis thaliana.

Front. Plant Sci. 7:360.

doi: 10.3389/fpls.2016.00360
The biosynthesis of chlorophylls and carotenoids and the assembly of thylakoid membranes are critical for the photoautotrophic growth of plants. Different factors are involved in these two processes. In recent years, members of the DnaJ-like zinc finger domain proteins have been found to take part in the biogenesis and/or the maintenance of plastids. One member of this family of proteins, PSA2, was recently found to localize to the thylakoid lumen and regulate the accumulation of photosystem I. In this study, we report that the silencing of PSA2 in Arabidopsis thaliana resulted in variegated leaves and retarded growth. Although both chlorophylls and total carotenoids decreased in the psa2 mutant, violaxanthin, and zeaxanthin accumulated in the mutant seedlings grown under growth condition. Lower levels of non-photochemical quenching and electron transport rate were also found in the psa2 mutant seedlings under growth condition compared with those of the wild-type plants, indicating an impaired capability to acclimate to normal light irradiance when PSA2 was silenced. Moreover, we also observed an abnormal assembly of grana thylakoids and poorly developed stroma thylakoids in psa2 chloroplasts. Taken together, our results demonstrate that PSA2 is a member of the DnaJ-like zinc finger domain protein family that affects light acclimation and chloroplast development.

Keywords: Arabidopsis thaliana, DnaJ-like, zinc finger, chloroplast, thylakoid, xanthophyll, light acclimation

\section{INTRODUCTION}

The biosynthesis of chlorophylls and carotenoids and the assembly of thylakoid membranes are key processes for the development of plastids and are critical for the photoautotrophic growth of plants (Mochizuki et al., 1996; Pogson and Albrecht, 2011; Pogson et al., 2015). Different factors, including enzymes, transcription factors, structural proteins, and chaperones, are found to regulate the biogenesis and functioning of chloroplasts through these two processes under different conditions (Albrecht et al., 2006; Aluru et al., 2006; Sakamoto et al., 2008; Stephenson et al., 2009; Pfalz and Pfannschmidt, 2013; Zhong et al., 2013). Among these factors, the involvement of the DnaJ-like zinc finger domain proteins has been revealed only in recent years (Brutnell et al., 1999; Ham et al., 2006; Lu S. et al., 2006; Shimada et al., 2007; Lu Y. et al., 2011; Whippo et al., 2011; Zhong et al., 2013). These proteins are characterized by a C-terminal $4 \times$ repetition of the $\mathrm{CxxCx}(\mathrm{G}) \mathrm{x}(\mathrm{G})$ (the glycine 
residues are not absolutely conserved) motif resembling the zinc finger domain of bacterial DnaJ proteins, but do not retain the J-domain of those DnaJ proteins (Preisigmuller and Kindl, 1993).

Brutnell et al. (1999) characterized the first member of this family, BUNDLE SHEATH DEFECTIVE2 (BSD2). As a stroma protein, BSD2 is required for the accumulation of ribulose-1,5-bisphosphate carboxylase/oxygenase (Rubisco) in maize (Brutnell et al., 1999). Further studies have demonstrated that other DnaJ-like zinc finger domain proteins are also involved in the function and development of plastids. For examples, ORANGE (OR) from cauliflower and its orthologs from other plant species are located in both the chloroplast and the nucleus and regulate the transition from non-pigmented plastid forms into carotenoid-accumulated ones (Lu S. et al., 2006; Kim et al., 2013; Sun et al., 2015; Zhou et al., 2015). LOW QUANTUM YIELD OF PHOTOSYSTEM II1 (LQY1) is a thylakoid protein that functions in the repair and assembly of photosystem II (PSII) under high irradiance (Lu, 2011; Lu Y. et al., 2011). Moreover, in tobacco, Tsip1 is found to physically associate with the chloroplast surface and can be recruited to the nucleus to promote the expression of stress-related genes (Ham et al., 2006).

In a recent study, another DnaJ-like zinc finger domain protein, PSA2, was identified in both maize and Arabidopsis thaliana as a thylakoid lumen protein that promotes the biogenesis of photosystem I (PSI) (Fristedt et al., 2014). The silencing of PSA2 resulted in severely retarded growth and pale green leaves, with increased photoinhibition due to the defect in PSI. The characterization of PSA2 provided novel insight to the function of the DnaJ-like zinc finger domain proteins in modulating the adaptation of chloroplasts to light stress.

In the present work, we studied the subcellular localization of PSA2, and compared the growth, pigment components, gene expression, chlorophyll fluorescence and chloroplast ultrastructure of the psa2 mutant and the wild-type (WT) A. thaliana seedlings. Our results showed that both light acclimation and chloroplast development are affected by the silencing of PSA2.

\section{MATERIALS AND METHODS}

\section{Plant Materials and Growth Conditions}

All A. thaliana plants, including the wild type (WT) and two mutants of PSA2 (At2g34860), psa2-1 (CS445540, i.e., GABI_475C12, with the T-DNA insertion at the $5^{\prime}$-UTR of PSA2) and psa2-2 (CS430127, i.e., GABI_314G07, with T-DNA insertion in the second intron), are of the Col-0 ecotype background. Seeds purchased from the Arabidopsis Biological Resource Center (ABRC, Ohio State University, Columbus, OH) were surfacesterilized and then plated onto Murashige and Skoog (MS) medium. After $3 \mathrm{~d}$ stratification at $4^{\circ} \mathrm{C}$ in the dark, seeds were germinated at $22-25^{\circ} \mathrm{C}$ under a light intensity of $100 \mu \mathrm{mol}$ photons $\mathrm{m}^{-2} \mathrm{~s}^{-1}$ with a $14 \mathrm{~h} / 10 \mathrm{~h}$ light/dark photoperiod. Twoweek-old seedlings were moved to grow in soil (a mixture of peat moss, vermiculite and perlite at 1:1:1) under the same conditions.

\section{Molecular Manipulation and Plant Transformation}

Genomic DNA was extracted from mature leaves (Murray and Thompson, 1980). Homozygous seedlings of the psa2-1 mutant and its progenies were screened according to the SIGnAL iSect tool $^{1}$ using three primers, i.e., a forward primer, LP, that binds from -610 bp upstream of the translation initiation codon (ATG) of PSA2, a reverse primer, RP, that binds from $300 \mathrm{bp}$ downstream of the ATG codon, and the primer 8409 targets the sequence of vector pAC106 that was originally used for generating the T-DNA insertion mutants. All primers used for polymerase chain reaction (PCR) in this study are listed in Supplement Table S1. Genomic DNA extracted from each individual seedling was used as a template. Homozygous progeny lines were used in this study.

Total RNA was isolated using the RNAiso Plus reagent (TaKaRa, Shiga, Japan) and was then reverse transcribed to a first strand cDNA pool using a PrimeScript 1st Strand cDNA Synthesis Kit (TaKaRa) according to the manufacturer's manuals. Gene expression was analyzed by quantitative real-time PCR (qPCR) in a Thermal Cycler Dice Real Time System TP800 (TaKaRa) using SYBR Premix ExTaq II (TaKaRa), following the manufacturer's instructions, and calculated according to the comparative $C_{\mathrm{T}}$ method (Schmittgen and Livak, 2008). For each sample, at least three biological replicates were analyzed, and each experiment was repeated three times. ACT2 (At3g18780) was used as a reference.

For the expression of a fusion protein of PSA2 with a C-terminal green fluorescent protein (GFP), the full-length open reading frame (ORF) of PSA2 was amplified from the first stand cDNA pool by primers PAS2-GFP-F and PSA2-GFP-R. The amplicon was electrophoresed, extracted from the gel using a SanPrep Column DNA Gel Extraction Kit (Sangon, Shanghai, China), digested with NcoI and SpeI (TaKaRa), and ligated into pCAMBIA1302 (CAMBIA, Canberra, Australia) that was digested with the same enzymes and purified. This generated the construct 35S:PSA2-GFP for the expression of the PSA2GFP fusion protein by protoplast transfection according to Yoo et al. (2007).

We also amplified a genomic DNA fragment ranging from $-2,000$ bp upstream of the translation initiation codon to $1,000 \mathrm{bp}$ downstream the stop codon of PSA2, using primers PSA2-FL-F and PSA2-FL-R with genomic DNA as a template. The amplicon was electrophoresed, extracted from the gel, digested with KpnI and SalI (TaKaRa), and ligated into pCAMBIA1300 (CAMBIA) that was digested and purified as described above. This generated the construct PSA2:PSA2 for genetic complementation. The construct was transferred into the Agrobacterium tumefaciens strain GV3101 by electroporation (Lu S. et al., 2006) for transformation of psa2-1 plants using the floral dip method (Clough and Bent, 1998).

For all PCR amplifications, high-fidelity PrimeStar DNA polymerase (TaKaRa) was used according to the manufacturer's instruction throughout this study.

\footnotetext{
${ }^{1}$ http://signal.salk.edu/tdnaprimers.2.html
} 


\section{Pigment Analysis}

Pigments were extracted from the leaves and analyzed by reversephase high-performance liquid chromatography (HPLC) using a Waters 2695 separation module with a Spherisorb ODS2 column $(5 \mu \mathrm{m}, 4.6 \mathrm{~mm} \times 250 \mathrm{~mm})$ and 2998 photodiode array detector (PDA) (Waters, Milford, MA) following the method of Li et al. (2001). Deuteroporphyrin was used as an internal standard.

\section{Chlorophyll Fluorescence Measurements}

Chlorophyll fluorescence was measured using a portable MINIPAM fluorometer (Walz, Effelrich, Germany). Before the measurements, leaves were dark-adapted for $20 \mathrm{~min}$. The minimal yield of fluorescence $\left(F_{o}\right)$ under measuring light $(650 \mathrm{~nm})$ was measured at a very low light intensity $(0.8 \mu \mathrm{mol}$ photons $\left.\mathrm{m}^{-2} \mathrm{~s}^{-1}\right)$. To estimate the maximum fluorescence yield $\left(F_{m}\right)$, a saturating pulse $\left(0.8 \mathrm{~s}, 5,000 \mu \mathrm{mol}\right.$ photons $\left.\mathrm{m}^{-2} \mathrm{~s}^{-1}\right)$ was applied. The ratio $F_{v} / F_{m}=\left(F_{m}-F_{o}\right) / F_{m}$ was calculated to indicate the maximum photochemical efficiency (Maxwell and Johnson, 2000). For the measurement of light-response curves of non-photochemical quenching (NPQ), electron transport rate (ETR) and PSII quantum yield (ФPSII), dark-adapted plants were illuminated at a series of photosynthetically active photon flux densities (PPFD) (0, 46, 96, 168, 257, 358, 535, 731, and $1,107 \mu \mathrm{mol}$ photons $\left.\mathrm{m}^{-2} \mathrm{~s}^{-1}\right)$. The duration of illumination at each light intensity was $3 \mathrm{~min}$, after which a saturation pulse was applied.

\section{Chloroplast Isolation, Fractionation, and Western Blot Analysis}

The isolation of intact chloroplasts and the preparation of stroma and thylakoid protein fractions were performed according to Hall et al. (2011). Fractionation of grana core, grana margin, and stroma lamellae was performed as described by Lu Y. et al. (2011).

Each fraction was suspended in sodium dodecyl sulfate (SDS) loading buffer (Green and Sambrook, 2012), denatured, and resolved by SDS-polyacrylamid gel electrophoresis (SDSPAGE). Proteins were semi-dry transferred to nitrocellulose membrane Protran BA 83 (GE Healthcare, Pittsburgh, PA, USA) for immunodetection. A peptide (CGLPNNKGLLRRPGA) was synthesized based on the putative amino acid sequence of PSA2 and used as an antigen to immunize rabbits by GenScript (Nanjing, China). Antibodies against LHCB2, $\mathrm{RbcL}$ and PsaA were purchased from Agrisera (Vännäs, Sweden). Horseradish peroxidase (HRP)-conjugated secondary antibody against rabbit IgG was from Promega (Madison, WI). Immobilon Western HRP substrate for chemiluminescent detection was from EMD Millipore (Darmstadt, Germany). Common protocols (Green and Sambrook, 2012) and the manufacturer's manuals for SDS-PAGE, semi-dry blotting and Western detection with chemiluminescent substrate were followed.

\section{Microscopy}

The 35S:PSA2-GFP-transfected protoplasts were cultivated in the dark for $12 \mathrm{~h}$ and then observed using a FLUOVIEW FV1000
Laser Confocal Microscopy System (Olympus, Tokyo, Japan) (Lee and Hwang, 2011). For transmission electron microscopy (TEM) analysis of chloroplast ultrastructure, leaves from 4-weekold seedlings were fixed, embedded and sectioned according to Faso et al. (2009). A Hitachi-7700 transmission electron microscope was used for observation and image capturing.

\section{Sequence Analysis}

The TAIR10 dataset of A. thaliana proteins was scanned using PatMatch ${ }^{2}$ for all proteins with the $4 \times$ repeats of the $\operatorname{CxxCx}(G) x(G)$ motifs (with at least one glycine residue in each motif). Proteins with this repeat at their C-termini were manually identified. Sequences of DnaJ-like zinc finger domain proteins from other plant species were downloaded from GenBank. Sequences were aligned using the ClustalX program and manually edited.

\section{Statistical Analysis}

Statistical significance was tested using GraphPad Prism6 (GraphPad Software). Data are shown as the mean \pm SE of at least three replications.

\section{RESULTS}

\section{PSA2 Belongs to the DnaJ-Like Zinc Finger Domain Protein Family}

Because DnaJ-like zinc finger domain proteins from different plants are found to regulate the development and function of plastids (Brutnell et al., 1999; Ham et al., 2006; Lu S. et al., 2006; Shimada et al., 2007; Lu Y. et al., 2011; Whippo et al., 2011; Zhong et al., 2013), we scanned the TAIR10 dataset of A. thaliana proteins, and identified a total of 10 homolog proteins that possess the $4 \times$ repeats of the $\operatorname{CxxCx}(G) x(G)$ motifs (with at least one glycine residue in each motif) at their C-terminal regions. Sequence comparison revealed that, in each protein, the four $\operatorname{CxxCx}(G) x(G)$ motifs are grouped into two pairs. In each pair, two motifs are separated by three amino acid residues which are not conserved. Six of these proteins (or their orthologs in other plant species) have been previously identified to have a chloroplast localization, including OR (At5g61670) (Lu S. et al., 2006), PSA2 (At2g34860) (Fristedt et al., 2014), LQY1 (At1g75690) (Lu, 2011; Lu Y. et al., 2011), BSD2 (At5g17840) (Brutnell et al., 1999), Tsip1 (At2g28460) (Ham et al., 2006), and pTAC5 (At4g13670) (Zhong et al., 2013) (Figure 1).

\section{psa2 Mutants Showed Pigment-Defective and Growth-Retarded Phenotypes}

We ordered T-DNA insertion lines of these ten genes encoding DnaJ-like zinc finger domain proteins from ABRC. For each gene, when available, at least three lines with different T-DNA insertion positions were used in this study. We first germinated all these mutant lines on MS plates without sucrose for two

\footnotetext{
${ }^{2}$ https://www.arabidopsis.org/cgi-bin/patmatch/nph-patmatch.pl
} 


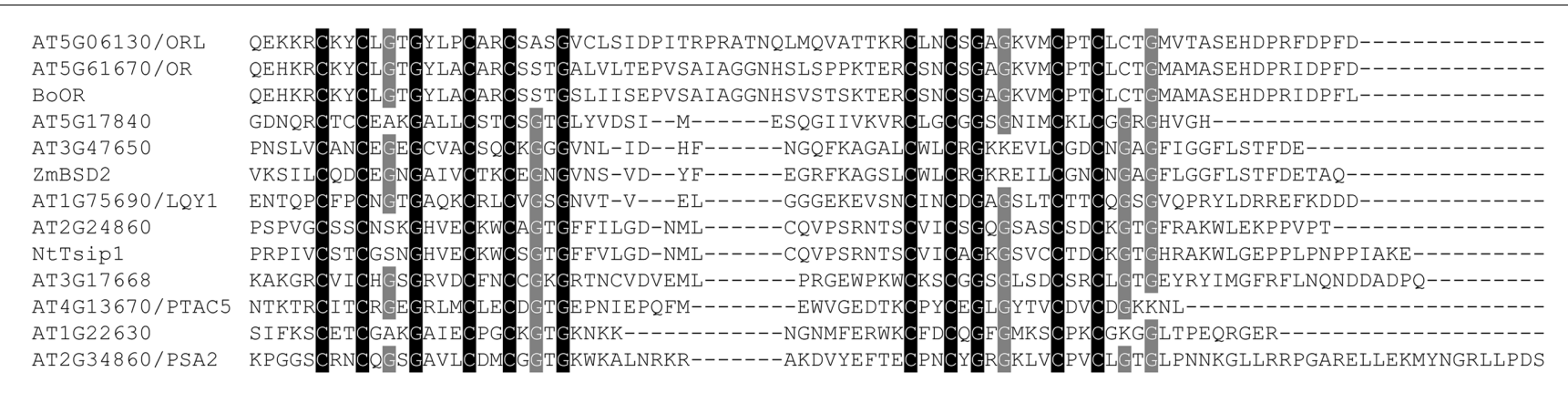

FIGURE 1 | Alignment of the C-terminal regions of DnaJ-like zinc finger domain proteins in Arabidopsis thaliana. Previously reported members from other plant species, including BSD2 from maize (ZmBSD2), OR from cauliflower (BoOR) and Tsip1 from tobacco (NtTsip1) were also incorporated. Sequences were aligned using ClustalX and manually edited. Conserved amino acid residues of the zinc finger domain were shaded.

weeks. For most of these lines, juvenile seedlings of the mutants did not show a distinct phenotype from the WT plants (data not shown). However, for PSA2, seedlings of the psa2-1 line with the T-DNA insertion at its $5^{\prime}$-UTR showed pale yellow leaves and severely retarded growth (Figure 2A). Another line, psa2-2, with the T-DNA insertion in the second intron died shortly after de-etiolation (data not shown). The expression level of PSA2 in psa2-1 was about the $17 \%$ of the WT level, whereas in psa2-2, it was undetectable (data not shown). When we then germinated these mutant lines on MS plates supplied with $1 \%$ sucrose, the psa2-1 seedlings showed variegated leaves and partially promoted growth (Figure 2B), whereas the psa2-2 line did not show significant recovery (data not shown). In the variegated leaves of the psa2-1 mutant, only the vein areas were green and other regions were pale yellow (Figure 2C). When we moved these psa2 mutant seedlings from MS plates with sucrose to the soil, only the psa2-1 plants survived, although they still had variegated leaves and retarded growth (Figure 2D). We repeated this for three batches, and observed identical results for each mutant line.

When flowering, the psa2-1 seedlings were much smaller than the WT plants (Figure 2G) and had thinner inflorescence branches and fewer flowers (Figure 2E). The juvenile siliques of the psa2-1 mutant were shorter than those of the WT plants, and they also had a pale yellow color (Figure 2F). All psa2-1 mutant seedlings had similar phenotypes at each developmental stage, and only representative pictures are presented in Figure 2.

We then selfed the psa2-1 line and harvested the $\mathrm{F}_{1}$ seeds. After germination, no phenotypic segregation was observed in the progeny population. All mature $\mathrm{F}_{1}$ plants showed variegated leaves, consistent with their parents. We randomly selected ten different $F_{1}$ plants, and our PCR analysis showed that all of these were homozygous (data not shown). We used a genetic fragment from $-2,000$ bp upstream of the translation initiation codon to $1,000 \mathrm{bp}$ downstream of the stop codon of PSA2 (PSA2:PSA2) to transform the psa2-1 plants. The transgenic progeny grew normally with green leaves, similar to the wildtype (Figure $2 \mathbf{H}$ ). This demonstrated that the disruption of the PSA2 gene is responsible for the observed phenotypes of the psa2 mutants.

\section{Tissue Specificity of the Expression of PSA2}

We compared the expression levels of PSA2 in different tissues. From our qPCR results, PSA2 was highly expressed in leaves, followed by flowers and siliques. The expression of PSA2 in both root and stems was relatively low (Figure 3).

\section{Subcellular Localization of PSA2}

Target $\mathrm{P}^{3}$ predicted a chloroplast localization of PSA2. This was confirmed by our protoplast transformation and confocal observation. Transient expression of the PSA2-GFP fusion protein showed a clear fluorescence signal in chloroplasts (Figure 4).

For a detailed understanding of PSA2 localization, we isolated intact chloroplasts from mature leaves of the WT plants and further lysed and centrifuged them to separate the supernatant (containing the outer and inner envelope membranes and stroma) and the pellet (containing mainly thylakoids). Our Western blots showed that PSA2 was only present in the pellet fraction, suggesting a thylakoid localization (Figure 5A). Furthermore, we separated the thylakoids into stroma lamellae and grana margin and grana core fractions by differential ultracentrifugation. Our Western blot results showed that PSA2 co-localized with PsaA, a component of the PSI reaction center, which is predominantly localized in SL fraction and could also be detected in grana margin (Figure 5B).

\section{psa2 Seedlings Accumulated Abnormal Levels of Chlorophylls and Carotenoids}

Because the psa2-1 leaves showed a variegated phenotype, we quantified the pigment contents in rosette leaves of 4-week-old seedlings by HPLC (Figure 6).

The amounts of different pigments are listed in Table 1. The chl $a$ and $b$ contents in psa2-1 were only 37 and $45 \%$ of the WT level, respectively. This resulted in a decrease of the chl $a / b$ ratio from 2.68 to 2.21 . The lutein, $\beta$-carotene, violaxanthin (Vx), and neoxanthin $(\mathrm{Nx})$ contents in the psa2-1 mutant were also lowered to approximately $30-40 \%$ of their corresponding WT

\footnotetext{
${ }^{3}$ http://www.cbs.dtu.dk/services/TargetP/
} 

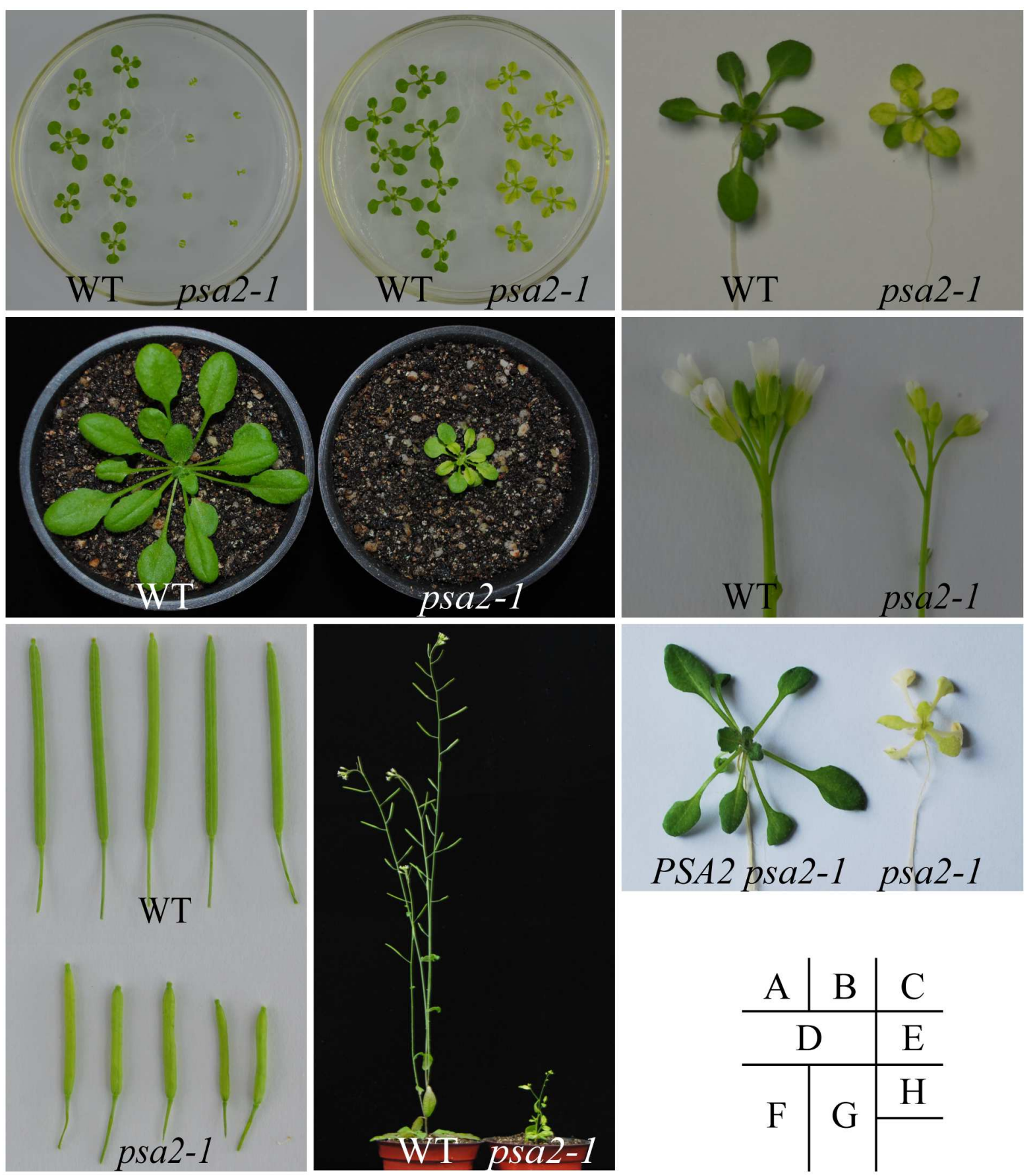

FIGURE 2 | Phenotype of the psa2-1 mutant plants. (A,B) psa2-1 seedlings germinated on MS plates without (A) or with (B) $1 \%$ sucrose for 2 weeks. (C) psa2-1 seedlings grown in soil for an additional 2 weeks after germination on MS plates. (D) A representative psa2-1 seedling grown in soil for 4 weeks after germination on MS plates. (E-G) Inflorescence (E), siliques (F), and mature plant (G) of the psa2-1 mutant. (H) Transformation with a wild type PSA2 gene fragment complemented the growth of psa2-1. Seedlings germinated on MS plates for 2 weeks were photographed.

levels. However, two carotenoids belonging to the xanthophyll cycle, zeaxanthin $(\mathrm{Zx})$, and antheraxanthin $(\mathrm{Ax})$, were found to accumulate in psa2-1 leaves but were not detectable in WT leaves. Overall, psa2-1 had only $41.29 \%$ carotenoids compared with the WT plants.
Because chlorophylls and total carotenoids were significantly lower in the psa2-1 mutant, we determined the expression levels of genes in the chlorophyll and carotenoid metabolic pathways. Our results demonstrated that PROTOCHLOROPHYLLIDE OXIDOREDUCTASE B (PORB), a key gene in chlorophyll 


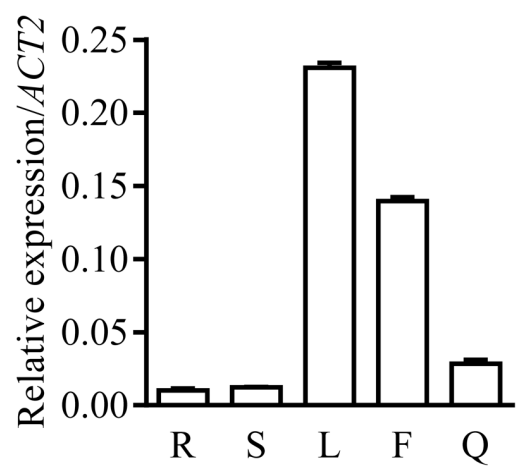

FIGURE 3 | Tissue specific expression of PSA2 in Arabidopsis thaliana. Transcript levels of $P S A 2$ in roots $(R)$, stems $(S)$, rosette leaves $(L)$, flowers $(F)$, and siliques $(\mathrm{Q})$ determined by quantitative real-time PCR are expressed relative to ACT2 (At3g18780). Data represent means $\pm \operatorname{SE}(n=6)$.

biosynthesis, was downregulated in the psa2-1 mutant to $39.7 \%$ of the WT level (Figure 7A), whereas two genes for chlorophyll turnover, CHLOROPHYLLASE1 and 2 (CLH1 and CLH2), were upregulated to 4.76- and 1.55-fold of the WT levels, respectively (Figure 7A). We further quantified the expression of genes for chlorophyll-binding proteins ( $L H C A$ and $L H C B$ ), only LHCB2 and $L H C B 4$ were significantly repressed in the psa2-1 mutant (Figure 7B).

For carotenoid metabolism, we did not observe a significant variation in the expression of most of the genes. ZEAXANTHIN EPOXIDASE (ZEP) and VIOLAXANTHIN DE-EPOXIDASE (VDE), two genes in the xanthophyll cycle, showed slight but significant upregulation to 1.41 - and 1.24-fold, respectively, of the WT levels (Figure 7C). The expression of NINE-CISEPOXYCAROTENOID DIOXYGENASE 3 (NCED3), the gene for carotenoid turnover, was downregulated to $29 \%$ of the WT level in the psa2-1 mutant (Figure 7C).

\section{PSA2 is Required for Chloroplast Development}

The variegated leaves of the psa2-1 mutant suggested a possible defect in chloroplast development. To this end, we sectioned leaves of the 4-week-old seedlings and observed them with TEM. In WT chloroplasts, approximately 10 layers of thylakoids were stacked in each grana, which were connected to each other by stroma lamellae. In each chloroplast, only a few starch grains and plastoglobules were observed (Figures 8A,B). In the green regions of the variegated psa2-1 leaves, approximately 30-50 layers of thylakoids were slantly piled and connected to other stacks from the margin. No distinct stroma lamellae were observed (Figures 8C,D). Dozens of plastoglobules were found in each chloroplast in this region (Figures $\mathbf{8 C , D}$ ). In the pale yellow regions of the mutant leaves, almost no thylakoid stacking was found in the chloroplast, and the stroma lamellae were abnormally assembled (Figures 8E,F).

\section{psa2 Mutants are Defective in Acclimating to Normal Growth Light}

To assess the impact of the low accumulation of photosynthetic and photoprotective pigments and the poor chloroplast development on photosynthetic activity, we performed noninvasive fluorometric analyses. Compared with the WT plants, the psa2-1 mutant had a significantly lower minimal fluorescence $\left(F_{o}\right)$, whereas its maximal fluorescence $\left(F_{m}\right)$ was similar to the

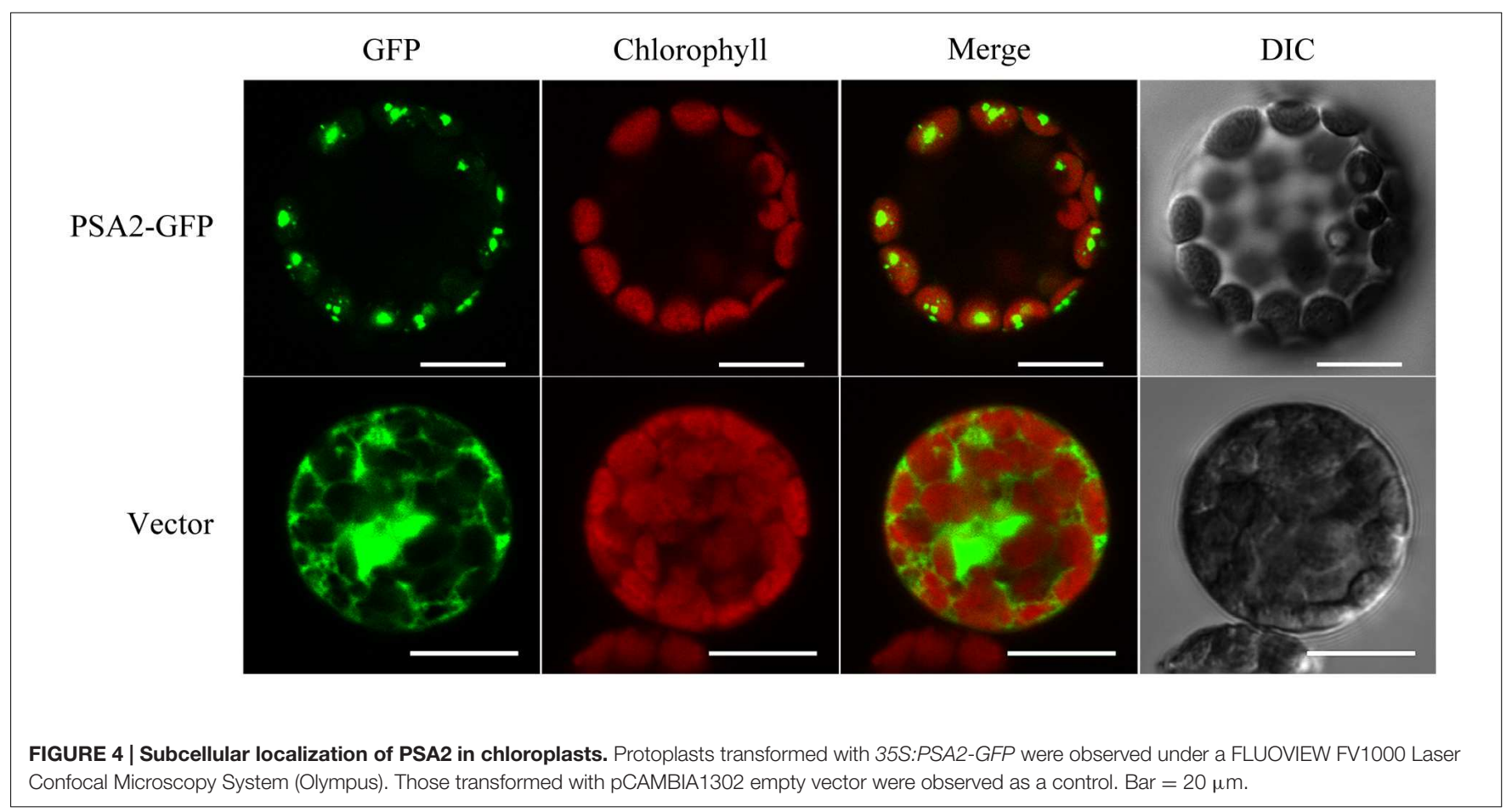


A

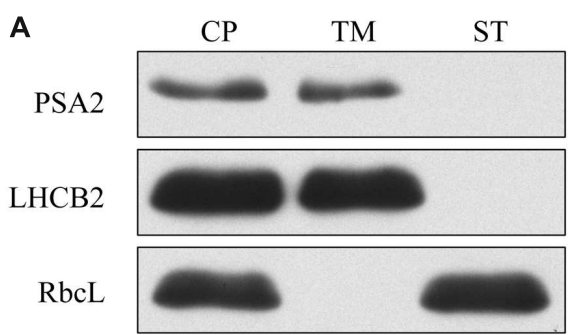

B

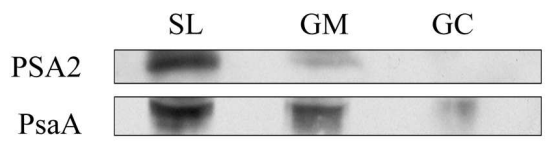

FIGURE 5 | Western blot showing that PSA2 localizes predominantly in stroma lamellae. (A) Purified chloroplasts (CP) were separated into the thylakoid membrane (TM) and stroma (ST) fractions, separated by SDS-PAGE, blotted and probed with the antiserum against PSA2. LHCB2 and RbcL were probed as controls. (B) Thylakoid membranes were furtherly subfractionated into stroma lamellae (SL), grana margin (GM), and grana core (GC) fractions, separated by SDS-PAGE, blotted, and probed with the antiserum against PSA2. PsaA was probed as a control.

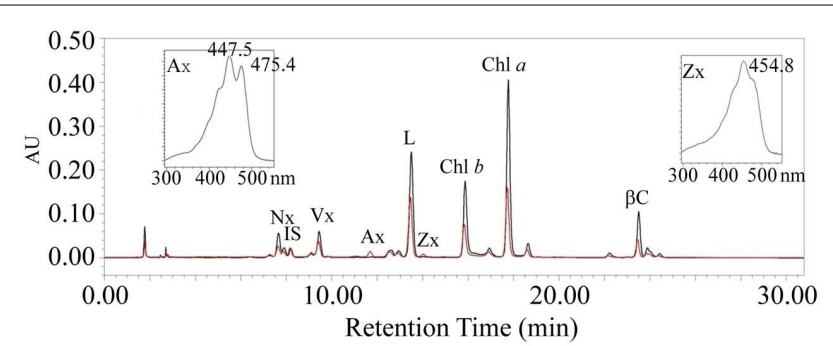

FIGURE 6 | High-performance liquid chromatography (HPLC) analysis of pigments in rosette leaves of the psa2-1 mutant (red) and the wild-type plants (black). Peaks are neoxanthin (Nx), internal standard (IS), violaxanthin $(\mathrm{Vx})$, antheraxanthin $(\mathrm{Ax})$, lutein $(\mathrm{L})$, zeaxanthin $(\mathrm{Zx}), \beta$-carotene $(\beta C)$, and chlorophylls (Chl a and $\mathrm{Chl} b)$. Insets are spectra profiles of $A x$ and Zx.

TABLE 1 | Leaf pigment profile of the Arabidopsis thaliana wild-type (WT) and psa2-1 mutant plants ( $\mu \mathrm{g} / \mathrm{g}$ fresh weight).

\begin{tabular}{lcc}
\hline & WT & psa2-1 \\
\hline Chl $a$ & $1036.60 \pm 85.44$ & $381.42 \pm 102.57^{* *}$ \\
Chl $b$ & $486.60 \pm 35.15$ & $216.96 \pm 49.00^{* *}$ \\
Chl $a / b$ & $2.14 \pm 0.31$ & $1.75 \pm 0.09^{* *}$ \\
& & \\
Lutein & $270.80 \pm 6.33$ & $111.97 \pm 1.88^{* *}$ \\
$\beta$-carotene & $97.73 \pm 7.66$ & $29.70 \pm 2.36^{* *}$ \\
Zeaxanthin & 0 & $8.23 \pm 2.66^{* *}$ \\
Antheraxanthin & 0 & $8.42 \pm 1.44^{* *}$ \\
Violaxanthin & $53.55 \pm 1.63$ & $19.47 \pm 0.64^{* *}$ \\
Neoxanthin & $58.92 \pm 2.36$ & $17.21 \pm 0.27^{* *}$ \\
\hline
\end{tabular}

Data represent means \pm SE (Student's $t$ test; $n=6 .{ }^{* *} P<0.01$ ).

WT level. This resulted in a significant reduction in the ratio of viable fluorescence $\left(F_{v}=F_{m}-F_{o}\right)$ to $F_{m}$, which is an indicator of the efficiency of PSII photochemistry, from 0.832 in the WT plant to 0.672 in psa2-1 (Table 2).

We then measured the light-responsive curves to assess NPQ, ETR and ФPSII. At $96 \mu \mathrm{mol}$ photons $\mathrm{m}^{-2} \mathrm{~s}^{-1}$, a light intensity close to the growth condition, NPQ of the psa2-1 plants was similar to that of the WT plants. However, above this light intensity, NPQ of the psa2-1 plants dropped significantly from its corresponding WT level (Figure 9A). Although the ETR of the WT plants increased with the increasing of actinic light intensity in our measurements, that of the psa2-1 seedlings dropped immediately when the light intensity was greater than $46 \mu \mathrm{mol}$ photons $\mathrm{m}^{-2} \mathrm{~s}^{-1}$. For example, when the light intensity reached $96 \mu \mathrm{mol}$ photons $\mathrm{m}^{-2} \mathrm{~s}^{-1}$, the ETR of psa2-1 was only $31.71 \%$ of the level in the WT plants (Figure 9B). The ФPSII of psa2-1 was always lower than that of the WT plants at all light intensities we measured, e.g., it was approximately $31.57 \%$ of the WT level at $96 \mu \mathrm{mol}$ photons $\mathrm{m}^{-2} \mathrm{~s}^{-1}$ and was almost abolished when the light intensity was greater than $196 \mu \mathrm{mol}$ photons $\mathrm{m}^{-2} \mathrm{~s}^{-1}$ (Figure 9C).

\section{DISCUSSION}

Previously, Fristedt et al. (2014) reported that PSA2 interacts with a protein complex that contains PsaG, and the psa2 mutants specifically lost the PSI core complex. In our study, we confirmed the PSA2-silencing phenotypes (retarded growth and pale yellow leaves) reported by Fristedt et al. (2014). However, when we supplied sucrose to the medium, the vein areas of the juvenile psa2-1 mutant leaves turned green, showing a promoted development of chloroplasts comparing with the pale yellow regions. Because the mutant line with the T-DNA insertion in the intron (psa2-2) was lethal when grown in soil, as previously reported and also found in our study, our work focused on the psa2-1 mutant as well.

In addition to the previously reported phenotypes, we observed an abnormal accumulation of $\mathrm{Zx}$ and $\mathrm{Ax}$ in leaves of the psa2-1 mutant. In plants, ZEP catalyzes the conversion from $\mathrm{Zx}$ to $\mathrm{Ax}$ and then to $\mathrm{Vx}$ in the light, and VDE catalyzes the reverse reaction in the dark. The conversions among these three carotenoid species form the xanthophyll cycle, which protects the photosynthetic apparatus (Demmig-Adams and Adams, 1996). Although Zx and Ax are usually undetectable in plants growing under normal growth light, they are found to accumulate under high light irradiance that inhibits ZEP but induces VDE (Chen and Gallie, 2012). The accumulation of $\mathrm{Zx}$ and $\mathrm{Ax}$ in psa2-1 plants suggests that the mutant is unable to acclimate to normal growth light. Moreover, psa2-1 had a higher $F_{o}$ and a lower $F_{v} / F_{m}$ compared with the WT plants, suggesting the occurrence of photoinhibitory damage under growth light. Because PSA2 is found to regulate the accumulation of PSI, the reduction of $F_{v} / F_{m}$ in $p s a 2-1$ is similar to the $p p d 1$ mutant, which also has impaired PSI biogenesis (Liu et al., 2012).

The activation of VDE and the impaired NPQ suggest a lower trans-thylakoid $\Delta \mathrm{pH}$ and an inhibited electron transport downstream of PSII, similar to the ppd1 mutant (Maxwell 


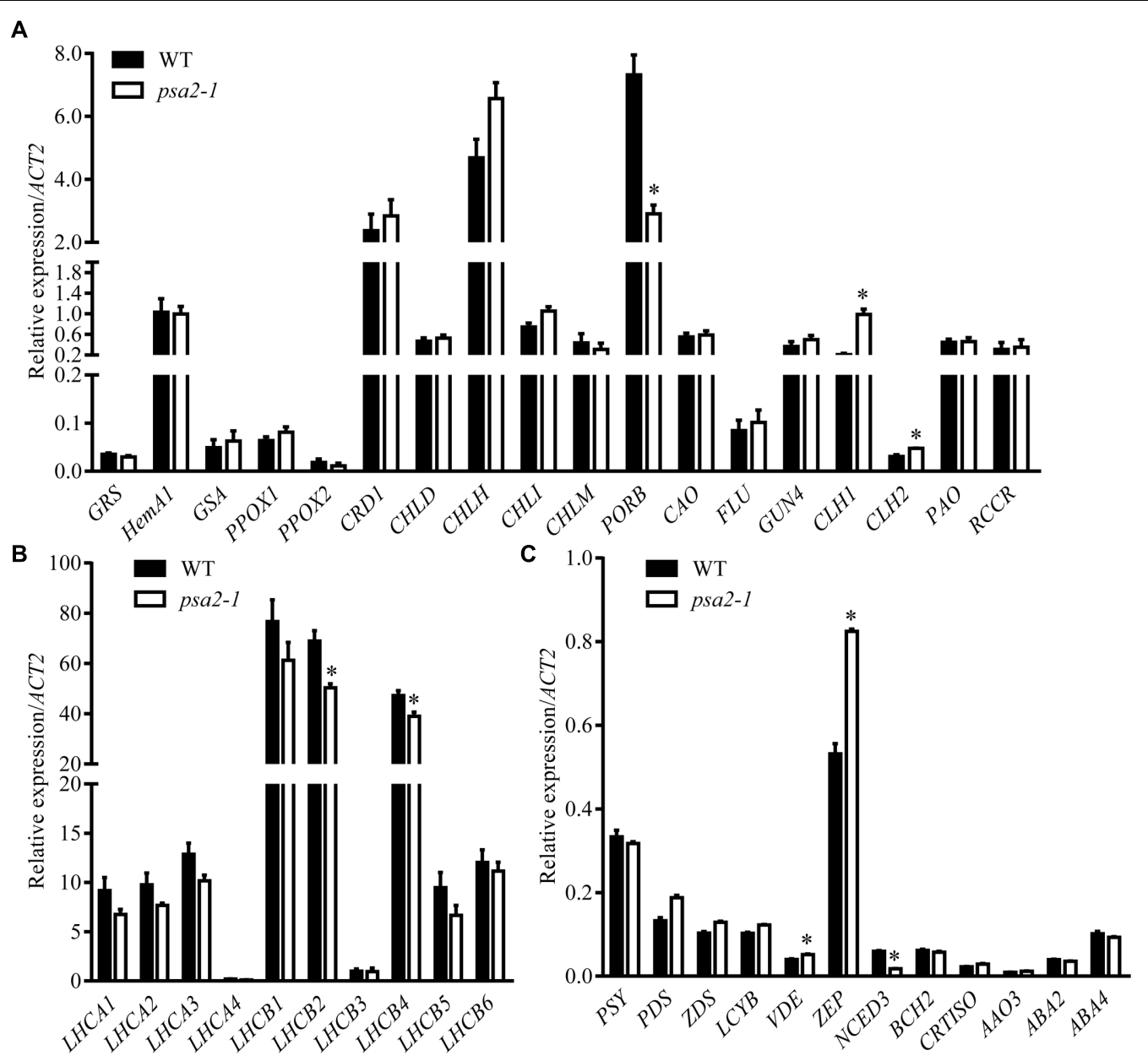

FIGURE 7 | Expression of the genes encoding enzymes for chlorophyll metabolism (A), chlorophyll binding proteins (B) and enzymes for carotenoid metabolism (C) in rosette leaves of the 4-week-old psa2-1 and wild-type (WT) seedlings. Transcript abundance of each gene was quantified by quantitative real-time PCR and are expressed relative to ACT2 (At3g18780). Data represent means \pm SE (Student's $t$ test; $n=6$. ${ }^{*} P<0.05$ ).

and Johnson, 2000; Liu et al., 2012). This was confirmed by our determination of the ETR and DPSII under a series of light intensities. The ETR of the psa2-1 plant dropped immediately when the light intensity was above $46 \mu \mathrm{mol}$ photons $\mathrm{m}^{-2} \mathrm{~s}^{-1}$. Considering that the psa2-1 mutant cannot effectively accumulate PSI, a lower ETR suggests that the photosynthetic electrons produced by PSII under growth light were not well accepted by PSI in psa2-1. As a result, ФPSII in psa2-1 also dropped rapidly and was approximately 0 under light intensities above $257 \mu \mathrm{mol}$ photons $\mathrm{m}^{-2} \mathrm{~s}^{-1}$, while the WT plants still retained substantial activity. These results are typical for mutants blocked in photosynthetic electron transport downstream of PSII, such as ppd1 (Meurer et al., 1996; Liu et al., 2012).

Besides the results of the carotenoid quantification and chlorophyll fluorescence, which support the conclusion of Fristedt et al. (2014), an important finding in our work is that PSA2 is essential for the assembly of thylakoid membranes.
Previous studies showed that the impairment of photosystems usually occurs with alternations in the chloroplast ultrastructure (Rock et al., 1992; Goss et al., 2007). For example, grana stacking in the thylakoid membranes is very important for the process of overall NPQ, and it has been reported that partial unstacking of the grana membranes strongly reduces the capacity for heat dissipation (Goss et al., 2007). In psa21, grana thylakoids were not observed in the pale yellow regions of the variegated leaves. Although there are more layers of thylakoids stacked in each grana in chloroplasts in the green regions, this may be a compensation for the defective organization of the macrostructure, which has yet to be determined but may be a result of the defective accumulation of PSI. A distinct difference between the ultrastructures of the WT and psa2-1 chloroplasts is the lack of inter-grana stroma lamellae.

In chloroplasts, PSII and light harvesting complex II are almost exclusively found in the stacked regions of the grana membranes, whereas PSI and light harvesting complex I are 

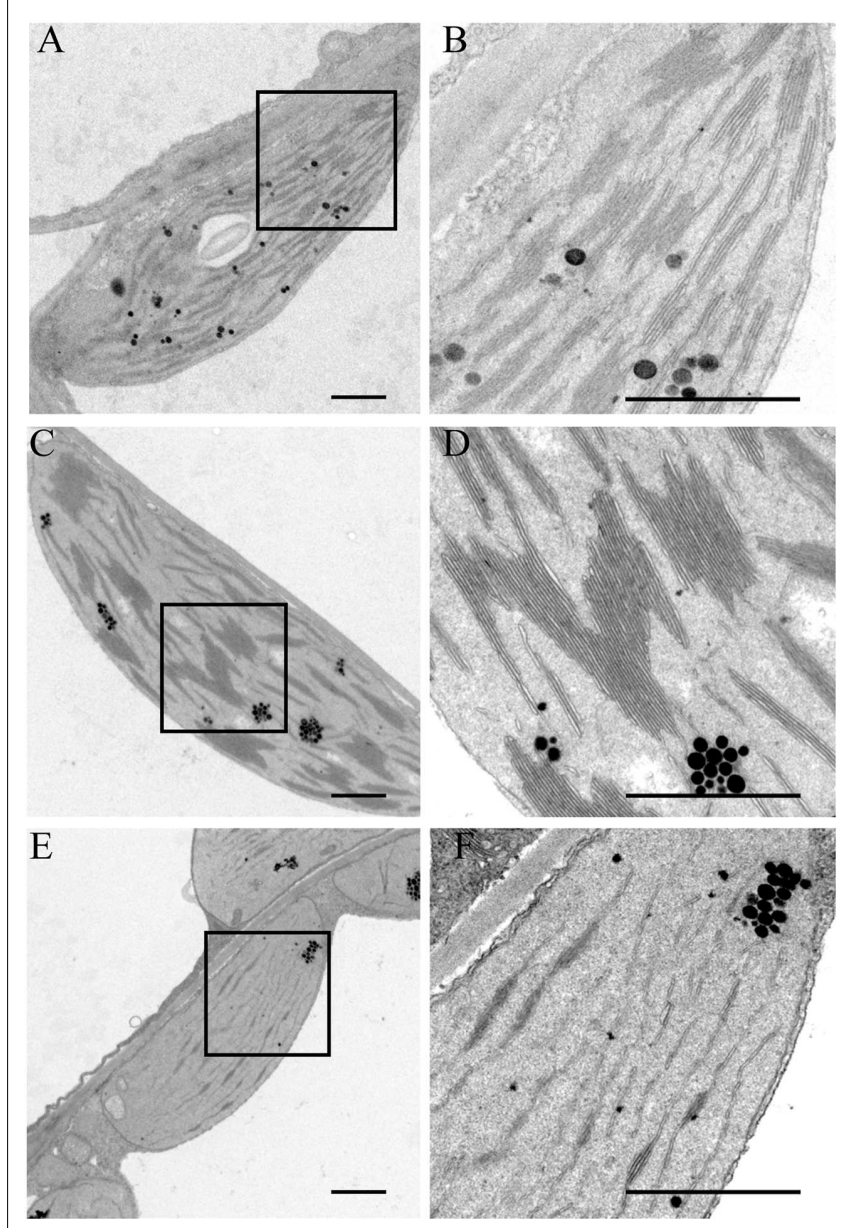

FIGURE 8 | Transmission electron microscopy (TEM) of chloroplasts from leaves of the wild-type plants $(A, B)$, and from the green (C,D) and pale yellow (E,F) regions of the variegated leaves of the psa2-1 seedlings. Bar $=1 \mu \mathrm{m}$. (B,D,F) are magnified view of the boxed regions in $(\mathbf{A}, \mathbf{C}, \mathbf{E})$, respectively.

TABLE 2 | Chlorophyll fluorescence parameters in the leaves of the wild-type (WT) and psa2-1 plants grown under growth light (100 $\mu \mathrm{mol}$ photons $\left.\mathrm{m}^{-2} \mathrm{~s}^{-1}\right)$.

\begin{tabular}{lcc}
\hline & WT & psa2-1 \\
\hline$F_{0}$ & $0.194 \pm 0.006$ & $0.382 \pm 0.060^{* *}$ \\
$F_{m}$ & $1.156 \pm 0.021$ & $1.167 \pm 0.204$ \\
$F_{v} / F_{m}$ & $0.832 \pm 0.007$ & $0.672 \pm 0.023^{* *}$
\end{tabular}

Data represent means $\pm S E$ (Student's $t$ test; $n=6$. ${ }^{* *} P<0.01$ ).

located in the stroma thylakoid membranes and are also found in the margin regions of the grana thylakoids (Goss et al., 2007). Our Western blot results showed that PSA2 colocalizes with PsaA, a component of PSI, in stroma thylakoid and, to a lesser degree, in grana margin. This subcellular localization of PSA2 helps to explain the function of this protein. Similar to PPD1, which also localizes to the same fraction and interacts with $\mathrm{PsaA} / \mathrm{PsaB}$ of PSI to regulate the assembly of PSI (Goss et al., 2007), PSA2 may be
A

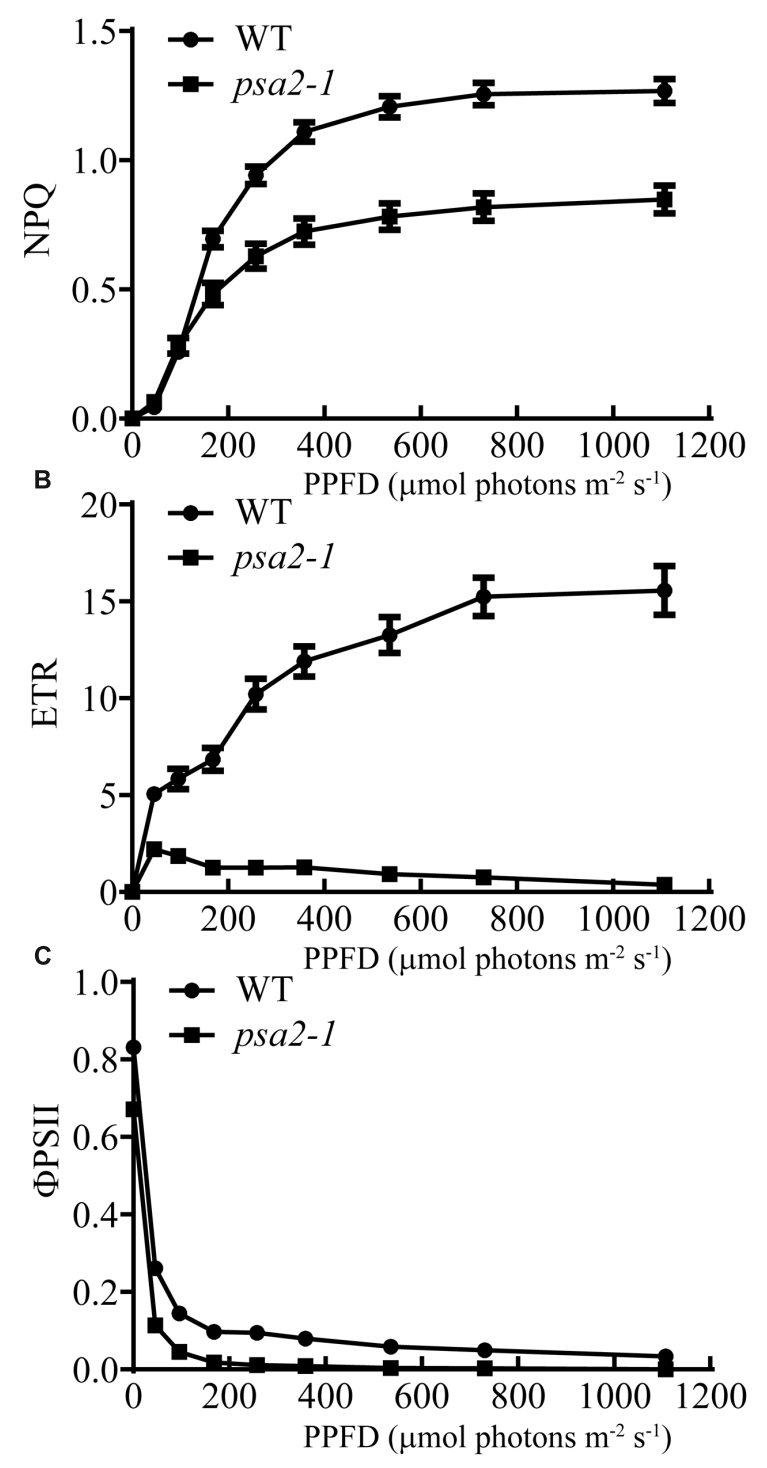

FIGURE 9 | Light-responsive curves of the non-photochemical quenching (NPQ) (A), photosynthetic electron transport rate (ETR) (B) and PSII quantum yield ( $\Phi$ PSII) (C) in the wild-type (WT) and psa2-1 seedlings. The measurements were taken at the following photosynthetically active photon flux densities (PPFD): 0, 46, 96, 168, 257, 358, 535, 731, and $1,107 \mu \mathrm{mol}$ photons $\mathrm{m}^{-2} \mathrm{~s}^{-1}$. Data represent means $\pm \mathrm{SE}(n=6)$.

involved in assembly of the thylakoids through its proteindisulfide isomerase activity; however, its mechanism remains unclear.

Taken together, the spectroscopy measurements, biochemical analyses and chloroplast ultrastructure observations demonstrate the impairments in the function of PSI and the assembly of thylakoid membranes in the psa2-1 mutant, thus providing a straightforward explanation for the pigment-deficient and growth-retarded phenotypes of this mutant. This work showed that PSA2 is a member of the DnaJ-like zinc finger domain 
protein family that is involved in the regulation of the function and development of the chloroplast.

\section{AUTHOR CONTRIBUTIONS}

Y-WW, S-MC, W-JW, X-QH, and ZZ performed experiments. C-FZ, ZZ, X-QH, and SL analyzed the data. S-MC and SL wrote the manuscript.

\section{FUNDING}

The work was supported by the State Key Basic Research and Development Plan of China (No. 2013CB127004), and the

\section{REFERENCES}

Albrecht, V., Ingenfeld, A., and Apel, K. (2006). Characterization of the snowy cotyledon 1 mutant of Arabidopsis thaliana: the impact of chloroplast elongation factor G on chloroplast development and plant vitality. Plant Mol. Biol. 60, 507-518. doi: 10.1007/s11103-005-4921-0

Aluru, M. R., Yu, F., Fu, A., and Rodermel, S. (2006). Arabidopsis variegation mutants: new insights into chloroplast biogenesis. J. Exp. Bot. 57, 1871-1881. doi: $10.1093 /$ jxb/erj008

Brutnell, T. P., Sawers, R. J., Mant, A., and Langdale, J. A. (1999). BUNDLE SHEATH DEFECTIVE2, a novel protein required for post-translational regulation of the $r b c L$ gene of maize. Plant Cell 11, 849-864. doi: 10.1105/tpc.11.5.849

Chen, Z., and Gallie, D. R. (2012). Violaxanthin de-epoxidase is ratelimiting for non-photochemical quenching under subsaturating light or during chilling in Arabidopsis. Plant Physiol. Biochem. 58, 66-82. doi: 10.1016/j.plaphy.2012.06.010

Clough, S. J., and Bent, A. F. (1998). Floral dip: a simplified method for Agrobacterium-mediated transformation of Arabidopsis thaliana. Plant J. 16, 735-743. doi: 10.1046/j.1365-313x.1998.00343.x

Demmig-Adams, B., and Adams, W. W. III (1996). The role of xanthophyll cycle carotenoids in the protection of photosynthesis. Trends Plant Sci. 1, 21-26. doi: 10.1016/S1360-1385(96)80019-7

Faso, C., Chen, Y. N., Tamura, K., Held, M., Zemelis, S., Marti, L., et al. (2009). A missense mutation in the Arabidopsis COPII coat protein Sec24A induces the formation of clusters of the endoplasmic reticulum and Golgi apparatus. Plant Cell 21, 3655-3671. doi: 10.1105/tpc. 109.068262

Fristedt, R., Williams-Carrier, R., Merchant, S. S., and Barkan, A. (2014). A thylakoid membrane protein harboring a DnaJ-type zinc finger domain is required for photosystem I accumulation in plants. J. Biol. Chem. 289, 30657-30667. doi: 10.1074/jbc.M114.587758

Goss, R., Oroszi, S., and Wilhelm, C. (2007). The importance of grana stacking for xanthophyll cycle-dependent NPQ in the thylakoid membranes of higher plants. Physiol. Plant. 131, 496-507. doi: 10.1111/j.1399-3054.2007.00964.x

Green, M. R., and Sambrook, J. (2012). Molecular Cloning: A Laboratory Manual, 4th Edn. New York, NY: Cold Springer Harbor Laboratory Press.

Hall, M., Mishra, Y., and Schröder, W. P. (2011). "Preparation of stroma, thylakoid membrane, and lumen fractions from Arabidopsis thaliana chloroplasts for proteomic analysis," in Chloroplast Research in Arabidopsis: Methods and Protocols, ed. R. P. Jarvis (New York, NY: Springer), 207-222.

Ham, B.-K., Park, J. M., Lee, S.-B., Kim, M. J., Lee, I.-J., Kim, K.-J., et al. (2006). Tobacco Tsip1, a DnaJ-type Zn finger protein, is recruited to and potentiates Tsi1-mediated transcriptional activation. Plant Cell 18, 2005-2020. doi: 10.1105/tpc.106.043158

Kim, S. H., Ahn, Y. O., Ahn, M.-J., Jeong, J. C., Lee, H.-S., and Kwak, S.-S. (2013). Cloning and characterization of an Orange gene that increases carotenoid accumulation and salt stress tolerance in transgenic sweetpotato cultures. Plant Physiol. Biochem. 70, 445-454. doi: 10.1016/j.plaphy.2013.06.011
National Natural Science Foundation of China (NSFC, Nos. 30771167 and 90817002).

\section{ACKNOWLEDGMENT}

We thank ABRC (Arabidopsis Biological Resource Center) for the Arabidopsis seeds.

\section{SUPPLEMENTARY MATERIAL}

The Supplementary Material for this article can be found online at: http://journal.frontiersin.org/article/10.3389/fpls.2016.00360

Lee, D. W., and Hwang, I. (2011). "Transient expression and analysis of chloroplast proteins in Arabidopsis protoplasts," in Chloroplast Research in Arabidopsis: Methods and Protocols, ed. R. P. Jarvis (New York, NY: Springer), 59-71.

Li, L., Paolillo, D. J., Parthasarathy, M. V., Dimuzio, E. M., and Garvin, D. F. (2001). A novel gene mutation that confers abnormal patterns of $\beta$-carotene accumulation in cauliflower (Brassica oleracea var. botrytis). Plant J. 26, 59-67. doi: 10.1046/j.1365-313x.2001.01008.x

Liu, J., Yang, H., Lu, Q., Wen, X., Chen, F., Peng, L., et al. (2012). PSBPDOMAIN PROTEIN1, a nuclear-encoded thylakoid lumenal protein, is essential for photosystem I assembly in Arabidopsis. Plant Cell 24, 4992-5006. doi: 10.1105/tpc.112.106542

Lu, S., Van Eck, J., Zhou, X., Lopez, A. B., O’Halloran, D. M., Cosman, K. M., et al. (2006). The cauliflower Or gene encodes a DnaJ cysteine-rich domaincontaining protein that mediates high levels of $\beta$-carotene accumulation. Plant Cell 18, 3594-3605. doi: 10.1105/tpc.106.046417

Lu, Y. (2011). The occurrence of a thylakoid-localized small zinc finger protein in land plants. Plant Signal. Behav. 6, 1881-1885. doi: 10.4161/psb.6.12.18022

Lu, Y., Hall, D. A., and Last, R. L. (2011). A small zinc finger thylakoid protein plays a role in maintenance of photosystem II in Arabidopsis thaliana. Plant Cell 23, 1861-1875. doi: 10.1105/tpc.111.085456

Maxwell, K., and Johnson, G. N. (2000). Chlorophyll fluorescence - a practical guide. J. Exp. Bot. 51, 659-668. doi: 10.1093/jexbot/51.345.659

Meurer, J., Meierhoff, K., and Westhoff, P. (1996). Isolation of high-chlorophyllfluorescence mutants of Arabidopsis thaliana and their characterisation by spectroscopy, immunoblotting and Northern hybridisation. Planta 198, 385396. doi: 10.1007/BF00620055

Mochizuki, N., Susek, R., and Chory, J. (1996). An intracellular signal transduction pathway between the chloroplast and nucleus is involved in de-etiolation. Plant Physiol. 112, 1465-1469. doi: 10.1104/pp.112.4.1465

Murray, M. G., and Thompson, W. F. (1980). Rapid isolation of high molecular weight plant DNA. Nucleic Acids Res. 8, 4321-4325. doi: 10.1093/nar/8.19.4321

Pfalz, J., and Pfannschmidt, T. (2013). Essential nucleoid proteins in early chloroplast development. Trends Plant Sci. 18, 186-194. doi: 10.1016/j.tplants.2012.11.003

Pogson, B. J., and Albrecht, V. (2011). Genetic dissection of chloroplast biogenesis and development: an overview. Plant Physiol. 155, 1545-1551. doi: 10.1104/pp.110.170365

Pogson, B. J., Ganguly, D., and Albrecht-Borth, V. (2015). Insights into chloroplast biogenesis and development. Biochim. Biophys. Acta 1847, 1017-1024. doi: 10.1016/j.bbabio.2015.02.003

Preisigmuller, R., and Kindl, H. (1993). Plant dnaJ homolog: molecular cloning, bacterial expression, and expression analysis in tissues of cucumber seedlings. Arch. Biochem. Biophys. 305, 30-37. doi: 10.1006/abbi.1993.1389

Rock, C. D., Bowlby, N. R., Hoffmann-Benning, S., and Zeevaart, J. A. (1992). The aba mutant of Arabidopsis thaliana (L.) Heynh. has reduced chlorophyll fluorescence yields and reduced thylakoid stacking. Plant Physiol. 100, 1796-1801. doi: 10.1104/pp.100.4.1796 
Sakamoto, W., Miyagishima, S. Y., and Jarvis, P. (2008). Chloroplast biogenesis: control of plastid development, protein import, division and inheritance. Arabidopsis Book 6, e0110. doi: 10.1199/tab.0110

Schmittgen, T. D., and Livak, K. J. (2008). Analyzing real-time PCR data by the comparative $C_{\mathrm{T}}$ method. Nat. Protoc. 3, 1101-1108. doi: 10.1038/nprot.2008.73

Shimada, H., Mochizuki, M., Ogura, K., Froehlich, J. E., Osteryoung, K. W., Shirano, Y., et al. (2007). Arabidopsis cotyledon-specific chloroplast biogenesis factor CYO1 is a protein disulfide isomerase. Plant Cell 19, 3157-3169. doi: 10.1105/tpc.107.051714

Stephenson, P. G., Fankhauser, C., and Terry, M. J. (2009). PIF3 is a repressor of chloroplast development. Proc. Natl. Acad. Sci. U.S.A. 106, 7654-7659. doi: 10.1073/pnas.0811684106

Sun, T. H., Zhou, F., Liu, C. J., Zhuang, Z., and Lu, S. (2015). The DnaJ-like zinc finger domain protein ORANGE localizes to the nucleus in etiolated cotyledons of Arabidopsis thaliana. Protoplasma doi: 10.1007/s00709-015-0919-x [Epub ahead of print].

Whippo, C. W., Khurana, P., Davis, P. A., DeBlasio, S. L., DeSloover, D., Staiger, C. J., et al. (2011). THRUMIN1 is a light-regulated actin-bundling protein involved in chloroplast motility. Curr. Biol. 21, 59-64. doi: 10.1016/j.cub.2010.11.059

Yoo, S. D., Cho, Y. H., and Sheen, J. (2007). Arabidopsis mesophyll protoplasts: a versatile cell system for transient gene expression analysis. Nat. Protoc. 2, 1565-1572. doi: 10.1038/nprot.2007.199
Zhong, L., Zhou, W., Wang, H., Ding, S., Lu, Q., Wen, X., et al. (2013). Chloroplast small heat shock protein HSP21 interacts with plastid nucleoid protein pTAC5 and is essential for chloroplast development in Arabidopsis under heat stress. Plant Cell 25, 2925-2943. doi: 10.1105/tpc.113. 111229

Zhou, X., Welsch, R., Yang, Y., Álvarez, D., Riediger, M., Yuan, H., et al. (2015). Arabidopsis OR proteins are the major posttranscriptional regulators of phytoene synthase in controlling carotenoid biosynthesis. Proc. Natl. Acad. Sci. U.S.A. 112, 3558-3563. doi: 10.1073/pnas.1420 831112

Conflict of Interest Statement: The authors declare that the research was conducted in the absence of any commercial or financial relationships that could be construed as a potential conflict of interest.

Copyright (c) 2016 Wang, Chen, Wang, Huang, Zhou, Zhuang and $\mathrm{Lu}$. This is an open-access article distributed under the terms of the Creative Commons Attribution License (CC BY). The use, distribution or reproduction in other forums is permitted, provided the original author(s) or licensor are credited and that the original publication in this journal is cited, in accordance with accepted academic practice. No use, distribution or reproduction is permitted which does not comply with these terms. 